c Servicio de Traumatología y Ortopedia Infantil, Complexo Hospitalario Universitario de A Coruña (CHUAC), SERGAS,

A Coruña, España

' Servicio de Radiología, Complexo Hospitalario

Universitario de A Coruña (CHUAC), SERGAS, A Coruña,

España

* Autor para correspondencia.

Correo electrónico: ana.maria.prado.carro@sergas.es

(A. Prado-Carro) https://doi.org/10.1016/j.anpedi.2017.03.016

1695-4033/

(c) 2017 Publicado por Elsevier España, S.L.U. en nombre de Asociación Española de Pediatría.

\section{Implicación de un brote nacional de infección por Serratia marcescens asociado a clorhexidina contaminada en un hospital pediátrico}

\section{Implication of a national outbreak of Serratia marcescens associated with a contaminated solution of chlorhexidine in a paediatric hospital}

\section{Sr. Editor:}

La ubicuidad de Serratia marcescens, su capacidad de sobrevivir en ambientes húmedos, así como de colonizar el tracto gastrointestinal y/o respiratorio, la hace responsable de múltiples brotes nosocomiales ${ }^{1}$, sobre todo en pacientes pediátricos, concretamente, recién nacidos, quienes sufren las peores consecuencias ${ }^{2,3}$. En ocasiones se localiza la fuente de infección, siendo de especial trascendencia, por sus consecuencias, aquellos brotes en los que está implicada una solución antiséptica ${ }^{3}$. En esta carta describimos la experiencia en un hospital infantil $(\mathrm{HI})$, en el contexto de un brote nacional por S. marcescens asociado al uso de un antiséptico de clorhexidina ( $\mathrm{CHX})$ contaminado ${ }^{4}$, entre agosto de 2014 y enero de 2015, con 148 casos (86 confirmados) en 10 CC.AA. Aunque la mayoría de los afectados tenían más de 65 años, en nuestro centro se vieron afectados 8 niños, entre el 22 de noviembre y 16 de diciembre de 2014. Todos desarrollaron bacteriemia con buena evolución, excepto un lactante que falleció en 24 horas tras el diagnóstico (letalidad 12,5\%).

Inicialmente, cuando aún se desconocía la extensión del brote a nivel nacional, se mantuvo la hipótesis de una fuente común y/o reservorio de $S$. marcescens, un medio líquido ubicado en el bloque quirúrgico del $\mathrm{HI}$, donde se había atendido a los 5 primeros pacientes afectados. La negatividad de los cultivos de los antisépticos con base de CHX utilizados en dicha unidad, el aislamiento de S. marcescens en una bolsa de concentrado de hematíes de una paciente transfundida en $\mathrm{UCl}$ pediátrica y no intervenida, hizo considerar el procedimiento de la transfusión como otra posible vía de transmisión de la infección, pues todos los casos habían sido transfundidos previamente al diagnóstico de la bacteriemia.

En el curso de la investigación, y tras la detección de 7 de los 8 casos, la Agencia Española del Medicamento comunicó la retirada de lotes de antisépticos de piel sana a base de $\mathrm{CHX}$ en solución acuosa o alcohólica (1-15, I-11, I-28, 1-29, $1-30,1-33$ e 1-35) comercializados en 2014 , a la vez que

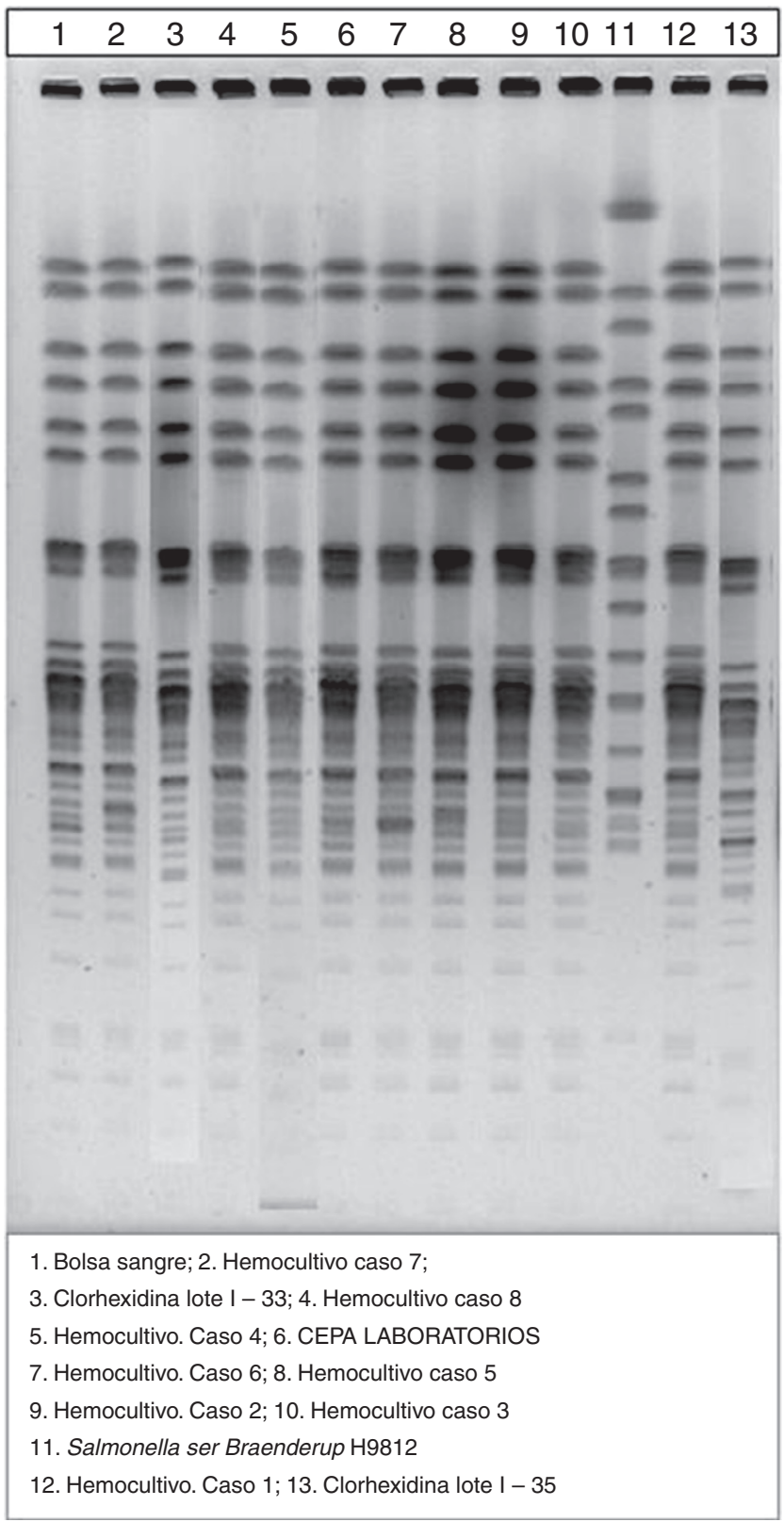

Figura 1 Patrones de electroforesis en campo pulsado de las cepas implicadas en el brote. 
desde el Servicio de Vigilancia Epidemiológica de Andalucía se nos alertaba del uso de dicho antiséptico en el $\mathrm{HI}$ como probable fuente del brote. Tras dicha notificación se comprobó que varios de los lotes implicados habían sido distribuidos en las unidades afectadas por el brote. A diferencia de otros estudios publicados en relación con este brote ${ }^{5}$, el estudio molecular realizado mediante técnica PFGE de las cepas aisladas en muestras de los pacientes, de las obtenidas en nuevas muestras $\mathrm{CHX}$ retiradas en el Hospital (lotes I-33 e 1-35) y de la cepa facilitada por los laboratorios que comercializaron el antiséptico contaminado demostró que todas las cepas pertenecían a un mismo clon (fig. 1).

Los procedimientos que con mayor probabilidad facilitaron la transmisión de la infección fueron aquellos en los que previamente se empleó dicho antiséptico, como: desinfección de piel previa al procedimiento quirúrgico e inserción de catéter y desinfección de llaves de 3 vías previas a la transfusión. Este hecho concuerda con lo referido en el informe del brote, donde el procedimiento médico asociado a la solución de $\mathrm{CHX}$ fue el uso de catéteres hasta en un $70 \%{ }^{4}$.

La rápida identificación y declaración del brote, la implementación de las medidas preventivas y el estudio molecular permitieron cerrar un brote epidémico que ha afectado a una población especialmente susceptible, como es la pediátrica. Es de destacar que en nuestro estudio se demuestra la identificación clonal de todos los aislados implicados en el brote con la cepa contaminante en origen y la afectación exclusiva de población pediátrica, que contó con un fallecimiento que pudo asociarse a la infección por $\mathrm{S}$. marcescens de los 148 pacientes afectados en el conjunto del territorio nacional. Consideramos esencial la mejora en las vías de comunicación entre agencias e instituciones sanitarias, dado que el problema se inició en agosto de 2014, persistiendo los brotes hasta diciembre del mismo año ${ }^{4}$.

\section{Agradecimientos}

A las Unidades de Cuidados Críticos y Urgencias, Pediatría General y Bloque quirúrgico del Hospital Infantil Virgen del Rocío, al Servicio de Vigilancia Epidemiológica de Andalucía y al Centro Regional de Transfusiones de Sevilla por su implicación en el brote e inestimable colaboración.

\section{Bibliografía}

1. Morillo A, González V, Aguayo J, Carreño C, Torres MJ, Jarana $D$, et al. A six-month Serratia marcescens outbreak in a neonatal intensive care unit. Enferm Infecc Microbiol Clin. 2016;34:645-51.

2. Voelz A, Müller A, Gillen J, Le C, Dresbach T, Engelhart S, et al. Outbreaks of Serratia marcescens in neonatal and pediatric intensive care units: Clinical aspects, risk factors and management. Int J Hyg Environ Health. 2010;213:79-87.

3. Villa J, Alba C, Barrado L, Sanz F, Gómez del Castillo E, Viedma E, et al. Long term evolution of multiple outbreaks of Serratia marcescens bacteremia in a neonatal intensive care unit. Pediatr Infect Dis J. 2012;31:1298-300.

4. Brote por Serratia marcescens asociado a la utilización de un antiséptico de clorhexidina contaminado. Madrid: Centro Nacional de Epidemiología, Instituto de Salud Carlos III. Boletín epidemiológico semanal. Semanas 19-22. 2016;24:85-101. [consultado 1 Mar 2017]. Disponible en: http://revista.isciii.es/ index.php/bes/article/view/988/1211

5. De Frutos $M$, López-Urrutia $L$, Domínguez-Gil $M$, Arias $M$, Muñoz-Bellido JL, Airosa JM, et al. Brote de Serratia marcescens producido por clorhexidina acuosa al $2 \%$ contaminada. Enferm Infecc Microbiol Clin. 2016, http://dx.doi.org/ 10.1016/j.eimc.2016.06.016

Áurea Morillo ${ }^{\mathrm{a}, *}$, María José Torres ${ }^{\mathrm{b}}$, María Teresa Alonso Salas ${ }^{c}$, Manuel Conde ${ }^{\mathrm{a}}$ y Javier Aznar ${ }^{\mathrm{a}, \mathrm{d}}$

a Unidad Clínica de Enfermedades Infecciosas, Microbiología y Medicina Preventiva, Hospital Universitario Virgen del Rocío, Sevilla, España

b Departamento de Microbiología, Universidad de Sevilla, Sevilla, España

c Unidad Clínica de Cuidados Críticos y Urgencias

Pediátricas, Hospital Universitario Virgen del Rocío, Sevilla, España

d Instituto de Biomedicina de Sevilla (IBiS), Hospital Universitario Virgen del Rocío, CSIC Universidad de Sevilla, Sevilla, España

* Autor para correspondencia.

Correo electrónico: aurea.morillo@gmail.com (Á. Morillo).

https://doi.org/10.1016/j.anpedi.2017.04.007

1695-4033/

(c) 2017 Publicado por Elsevier España, S.L.U. en nombre de Asociación Española de Pediatría. 